Received: 10 July 2017

Accepted: 4 October 2017

Published online: 17 October 2017
ENTIFIC REP

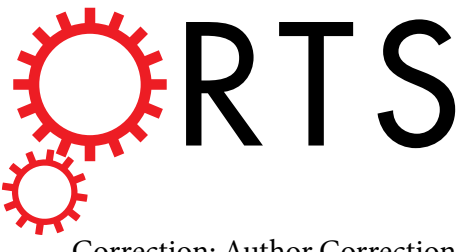

Correction: Author Correction

\section{Computational Analysis of} the Molecular Mechanism of RamR Mutations Contributing to Antimicrobial Resistance in
Salmonella enterica

Yen-Yi Liu ${ }^{1} \&$ Chih-Chieh Chen $\mathbb{D}^{2,3,4,5}$

Antimicrobial resistance (AMR) in pathogenic microorganisms with multidrug resistance (MDR) constitutes a severe threat to human health. A major causative mechanism of AMR is mediated through the multidrug efflux pump (MEP). The resistance-nodulation-division superfamily (RND family) of Gramnegative bacteria is usually the major cause of MDR in clinical studies. In Salmonella enterica, the RND pump is translated from the acrAB gene, which is regulated by the activator RamA. Many MEP-caused AMR strains have high ramA gene expression due to mutations in RamR, which has a homodimeric structure comprising the dimerization domain and DNA-binding domain (DBD). Three mutations on the dimerization domain, namely Y59H, M84I, and E160D, are far from the DBD; the molecular mechanism through which they influence RamR's binding affinity to the ramA gene promoter and consequently disrupt RamA remains unclear. The present study conducted molecular dynamics simulations, binding free energy calculations, and normal mode analysis to investigate the mechanism through which $\mathrm{Y} 59 \mathrm{H}, \mathrm{M} 84 \mathrm{l}$, and E160D mutations on the dimerization domain influence the binding affinity of RamR to the ramA promoter. The present results suggest that the three mutations alter the RamR structure, resulting in decreased DNA-binding affinity.

In recent years, antimicrobial resistance (AMR) has become a global concern. Pathogens with AMR can threaten human life; if pathogens with AMR infect inpatients, their treatment periods tend to be prolonged, thereby increasing healthcare costs. The abuses of antimicrobial drugs in clinics and agricultural settings imperceptibly culture many AMR strains in nature. For example, carbapenem-resistant Klebsiella pneumonia ${ }^{1}$ and colistin-resistant Enterobacteriaceae ${ }^{2}$ can pose severe threats to human life.

The mechanism of AMR includes target alteration, drug inactivation, decreased permeability, and increased efflux $^{3}$. Multidrug resistance (MDR) is an important aspect of AMR, and the multidrug efflux pump (MEP) ${ }^{4}$ is one of the essential mechanisms that mediate MDR. Several MEP-caused MDR pathogens include Salmonella enterica ${ }^{5}$, Escherichia coli ${ }^{6}$, Staphylococcus aureus ${ }^{7}$, and K. pneumonia ${ }^{8}$. The bacterial MEP can be classified into five families ${ }^{9}$ : resistance-nodulation-division superfamily (RND family) ${ }^{10}$, major facilitator superfamily ${ }^{11}$, ATP-binding cassette superfamily ${ }^{12}$, small MDR family ${ }^{13}$, and multidrug and toxic compound extrusion family ${ }^{14}$. Of the five MEP families, the RND family is the major cause of MDR in clinical studies ${ }^{15}$; for instance, the $a c r A B$ gene in E. coli and S. enterica ${ }^{16}$. Several studies on RND family-caused bacterial MDR ${ }^{17,18}$ have reported that RamA, the activator of the $a c r A B$ gene, is an essential target for elucidating the MDR mechanism, particularly in S. enterica ${ }^{19}$ and K. pneumonia ${ }^{20,21}$. In 2008, Abouzeed et al. ${ }^{22}$ identified ramR, a TetR family of transcriptional repressor gene, which is located upstream of the $\operatorname{ram} A$ gene. RamR binds to the promoter region of the $\operatorname{ram} A$

${ }^{1}$ Institute of Population Health Sciences, National Health Research Institutes, Miaoli, 35053, Taiwan. ${ }^{2}$ Institute of Medical Science and Technology, National Sun Yat-sen University, Kaohsiung, 80424, Taiwan. ${ }^{3}$ Medical Science and Technology Center, National Sun Yat-sen University, Kaohsiung, 80424, Taiwan. ${ }^{4}$ Rapid Screening Research Center, National Sun Yat-sen University, Kaohsiung, 80424, Taiwan. ${ }^{5}$ General Institute of Clinical Medicine, Kaohsiung Medical University, Kaohsiung, 80708, Taiwan. Correspondence and requests for materials should be addressed to C.-C.C. (email: chieh@imst.nsysu.edu.tw) 
gene to repress ramA gene transcription, thereby resulting in MDR. Several studies have reported different RamR mutations in the MDR strains of S. enterica and K. pneumonia that can reduce the repression of the ramA gene ${ }^{22-24}$. However, the molecular mechanism through which mutations, such as Y59H, M84I, and E160D ${ }^{22,25,26}$, on the dimerization domain of RamR reduce its ability to repress $r a m A$ gene expression remains unclear. The present study conducted molecular dynamics (MD) simulations and binding free energy calculations to investigate the mechanism through which Y59H, M84I, and E160D mutations in the dimerization domain cause MDR by assessing the binding events between the RamR protein and the ramA promoter.

We constructed four structures, specifically, the wild-type RamR (WT-RamR) and three mutants (MTs), namely Y59H-RamR, M84I-RamR, and E160D-RamR. The root mean square deviation (RMSD) and RMS fluctuation (RMSF) simulation results suggested that mutations in the dimerization domain of RamR might stabilize the overall structure but make structural changes in the DNA-binding domain (DBD), which could influence RamR's binding affinity to the ramA promoter DNA. To further investigate the mutation-induced changes in interaction properties, the total free energy of the MT and WT complexes was calculated to obtain the binding free energy. In addition, normal mode analysis (NMA) was performed to determine whether the mutations alter the dynamics networks to influence large-scale domain motion. The binding free energy calculations and NMA results were in accordance with the observations from MD simulations.

\section{Materials and Methods}

Three-dimensional models of MT-RamR structures. We selected $3 \mathrm{VVX}^{27}$ as the template to run MODELLER (9v8) ${ }^{28}$ for predicting the structures of Y59H-, M84I-, and E160D-RamR. Three mutant RamR models were constructed on the basis of the sequence and structural alignment of the $3 \mathrm{VVX}$ template. The qualities of all modeled structures were assessed through Ramachandran plots ${ }^{29}$. The steepest descent algorithm ${ }^{30}$ was $^{-}$ used for structural optimization (energy minimization), and $\mathrm{PyMOL}^{31}$ was used for all three-dimensional (3D) structures and charge distribution presentation.

Model of the RamR-DNA complex structure. Because the structure of the RamR-DNA complex is yet to be solved, we predicted its structure. Previous studies have demonstrated that RamR acts as a repressor and binds to the promoter region (TATAATGAGTGCTTACTCACTCATAATC) of the ramA gene to inhibit ramA gene expression ${ }^{32}$. Therefore, we used 3D-DART ${ }^{33}$ to construct a 3D model of the ramA promoter. Furthermore,

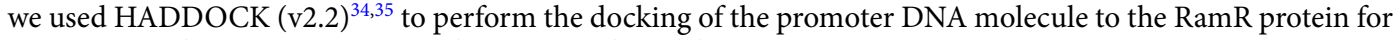
constructing the RamR-DNA complex structural model.

Molecular dynamics simulation. Molecular dynamics (MD) simulations were performed using GROMACS software (ver. 5.1.4) ${ }^{36}$. The Amber99 force field was used for energy calculations. The models were solvated with TIP4P water molecules and simulated in a cubic box with periodic boundary conditions. The energy of the models was first minimized using the steepest descent algorithm. The simulations were performed in the canonical NVT (constant number of particles, volume, and temperature) and NPT (constant number of particles, pressure, and temperature) ensembles through the position-restrained MD simulation for $100 \mathrm{ps,} \mathrm{respectively.}$ The linear constraint solver algorithm was used to constrain the bonds ${ }^{37}$. The MD simulations were performed at constant pressure and temperature for $20 \mathrm{~ns}$ using an integration time step of $2 \mathrm{fs}$. The nonbonded interactions were cutoff at $10 \AA$. The coordinates from the MD simulations were saved at every 100 time steps.

Trajectory analysis. To understand the structural and functional implications of the amino acid substitutions in the dimerization domain on the DBD, the trajectories of the WT and MT structures were analyzed for several structural properties as a function of time: (A) The RMSD of the C $\alpha$ atoms of the DBD with respect to the starting conformation, $r^{r e f}$, was calculated as $R M S D(t)=\left[\frac{1}{M} \sum_{i=1}^{N} m_{i}\left|r_{i}(t)-r_{i}^{r e f}\right|^{2}\right]^{1 / 2}$ where $M=\sum_{i} m_{i}$ and $r_{i}(t)$ is the position of atom $i$ at time $t$ after last square fitting of the structure to the reference structure; (B) the RMSF of the backbone $\mathrm{C} \alpha$ atoms with respect to the starting conformation was calculated as follows. The RMSF is a measure of the deviation between the position of particle $i$ and some reference position: $R M S F_{i}=\left[\frac{1}{T} \sum_{t_{j}=1}^{T}\left|r_{i}\left(t_{j}\right)-r_{i}^{r e f}\right|^{2}\right]^{1 / 2}$ where $T$ is the time over which one wants to average and $r_{i}^{r e f}$ is the reference position of particle $i$. Typically, this reference position will be the time-averaged position of the same particle, that is, $r_{i}^{r e f}=\bar{r}_{i}$; (C) the Debye-Waller factor (B-factor), which reflects the fluctuation of an atom about its average positions; each C $\alpha$ atom was calculated from the last $5 \mathrm{~ns}$ of the MD trajectories. The B-factor was calculated from the average RMSF per residue by using the relation $B-$ factor $=\frac{8}{3} \times \pi^{2} \times R M S F^{2}$. A large B-factor indicates a high flexibility of the atom.

Binding free energy calculation. The pmx tool was used to construct the hybrid topologies of the system used in the free energy calculations ${ }^{38,39}$. This tool automatically generates hybrid structures and topologies for amino acid mutations that represent the two physical states of the system. After obtaining this hybrid structure, free energy simulations were performed using GROMACS. From the 10-ns equilibrated trajectories, 100 snapshots were extracted, and a rapid 100-ps simulation was performed starting from each frame. The lambda $(\lambda)$ ranged from 0 to 1 and from 1 to 0 for the forward and backward integrations, respectively, thus describing the interconversion of the WT to MT systems and the MT to WT systems, respectively. Finally, the pmx tool was used to integrate over the multiple curves and subsequently estimate free energy differences using the fast-growth thermodynamic integration approach, which relies on the Crooks-Gaussian Intersection (CGI) protocol ${ }^{40}$. The $\Delta \mathrm{G}$ values for the mutations in the DNA-free and DNA-bound structures were calculated to construct a double free energy difference $(\Delta \Delta \mathrm{G})$. The final $\Delta \Delta \mathrm{G}$ values indicate whether mutations stabilize or destabilize the protein in the DNA-bound state. 


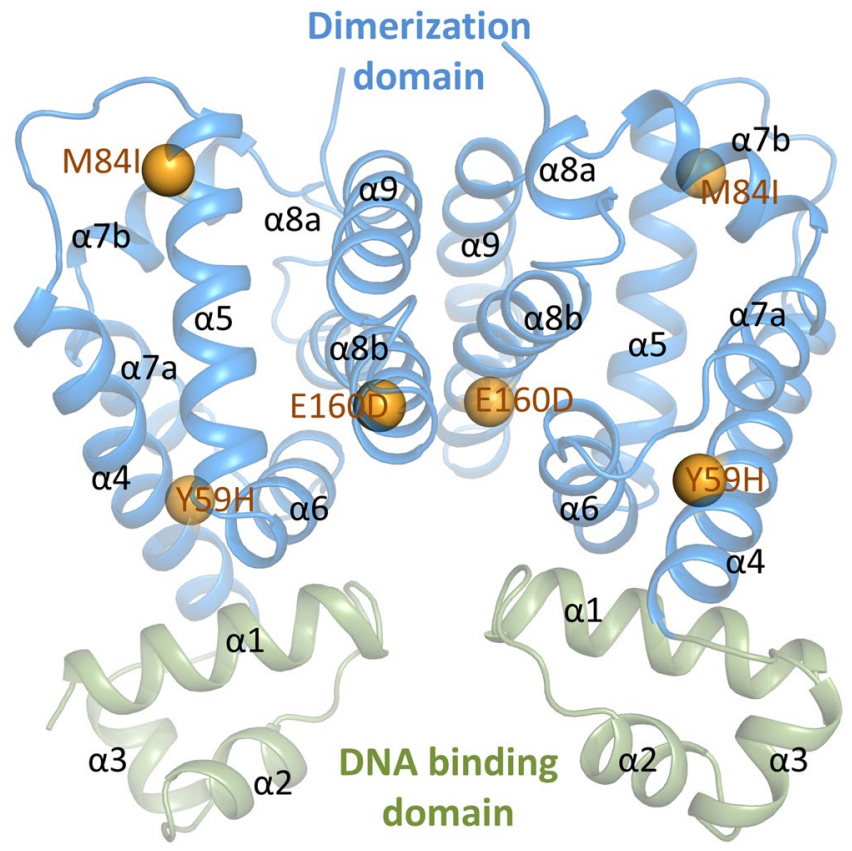

Figure 1. Protein structure of the human RamR dimer. The structure contains the DBD (green) and the dimerization domain (blue). The RamR mutations of Y59H ( $\alpha 4)$, M84I ( $\alpha 5)$, and E160D ( $\alpha 8 \mathrm{~b})$ are highlighted in orange spheres. The structural representation is generated by PyMOL.

Normal mode analysis. Normal mode analysis (NMA) is commonly adopted to estimate dynamics based on structures ${ }^{41-44}$. A low-frequency large-scale motion usually reflects real-world biological functional motions ${ }^{41,45}$. Although NMA is powerful, it is very time-consuming. In this study, we used Elastic Network Model $(\mathrm{ENM})^{46-48}$, a coarse-grained NMA method, to compute the low-frequency motions of the RamR protein using the web-service DynOmics ${ }^{49}$. In addition, pair-wise residue correlation maps were constructed.

\section{Results}

Predicted MT-RamR structures. The MT-RamR models were constructed using the published RamR crystal structure (Protein Data Bank Id: 3VVX) from S. typhimurium as the template; it is an all-alpha helix structure consisting of two dimerization domains and two DBDs (Fig. 1). The substitution residues, namely Y59, M84, and E160, are all located at the dimerization domain. Y59 is located at the boundary of the dimerization domain and the DBD; M84 is at the top of the dimerization domain; and E160 is at the dimerization surface between the two monomers. Figure 1 shows all amino acid substitutions in the RamR structure. One of the main purposes of the present study was to thoroughly examine the structural flexibility of the DBD with respect to the amino acid substitutions on the dimerization domain.

Predicted RamR-DNA complex structures. To construct the RamR-DNA complex structures, we first used 3D-DART ${ }^{33}$ to build the 3D coordinates of the RamR binding promoter (TATAATGAGTGCTTACTC ACTCATAATC), followed by the HADDOCK ${ }^{34,35}$ service tool. Figure $2 \mathrm{~A}$ presents the predicted complex model. On the basis of the study of Yamasaki et al. ${ }^{27}$, the fragment from residues 2 to 42 of RamR was defined as the DBD region. In our predicted complex structure, residues A31, V32, A34, R35, A37, G38, V39, E41, T43, and R46 in both chain A and chain B were involved in DNA-binding. Figure 2B shows a close view of the DNA-binding surface.

RMSD analysis of the DBDs of the WT- and MT-RamR structures. The trajectories from MD simulations of the WT- and MT-RamR-DBDs in the explicit solvent were calculated. The RMSD values of the C $\alpha$ atoms for WT- and MT-RamR-DBDs with regard to the initial structures were plotted (Fig. 3). The quality and convergence of the simulated dynamics trajectories can be estimated by assessing the RMSD values. For the simulations of the WT- and MT-RamR-DBDs, the obtained data demonstrated that after a rapid increase during the first $0.2 \mathrm{~ns}$, the trajectories were stable with the average values of $2.22,1.96$, and $2.32 \AA$ for WT-, M84I-, and E160D-RamR-DBDs, respectively, except for Y59H-RamR-DBD. The RMSD profiles of the DBDs with respect to the initial conformation of WT and MT structures during the course of the simulations were calculated. The RMSD values showed a large increase as a function of time for the Y59H-RamR-DBD compared with the WT-RamR-DBD. For the M84I- and E160D-RamR-DBDs, the RMSD variations were nearly similar to those of the WT-RamR-DBD (Fig. 3).

Residue fluctuation of the WT and MT RamR structures. The overall trend of the RMSF profiles of Y59H-, M84I-, and E160D-RamR was similar to that of WT-RamR, except for their DBDs, which exhibited different values than that of WT-RamR (Figure S1). We calculated the differences in RMSF values between MT- and 
(A)

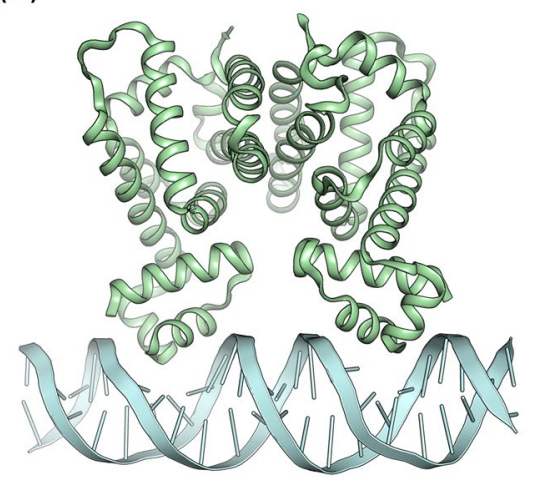

(B)

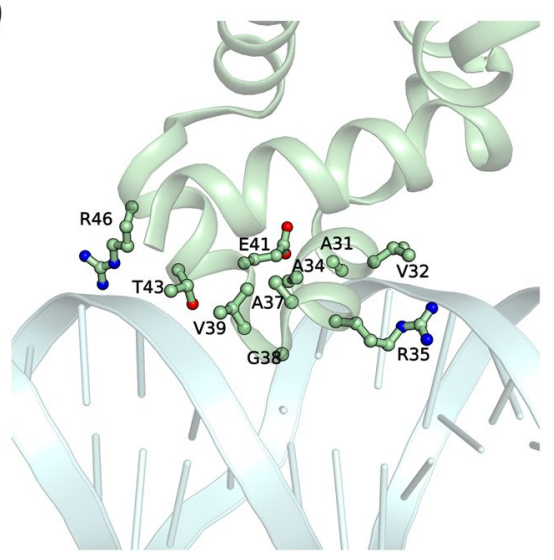

Figure 2. Ribbon representation of the RamR-DNA complex model structure. (A) The structure of the RamR (green)-DNA (cyans) complex, obtained through HADDOCK prediction. (B) A close view of the human RamR binding residues with DNA.

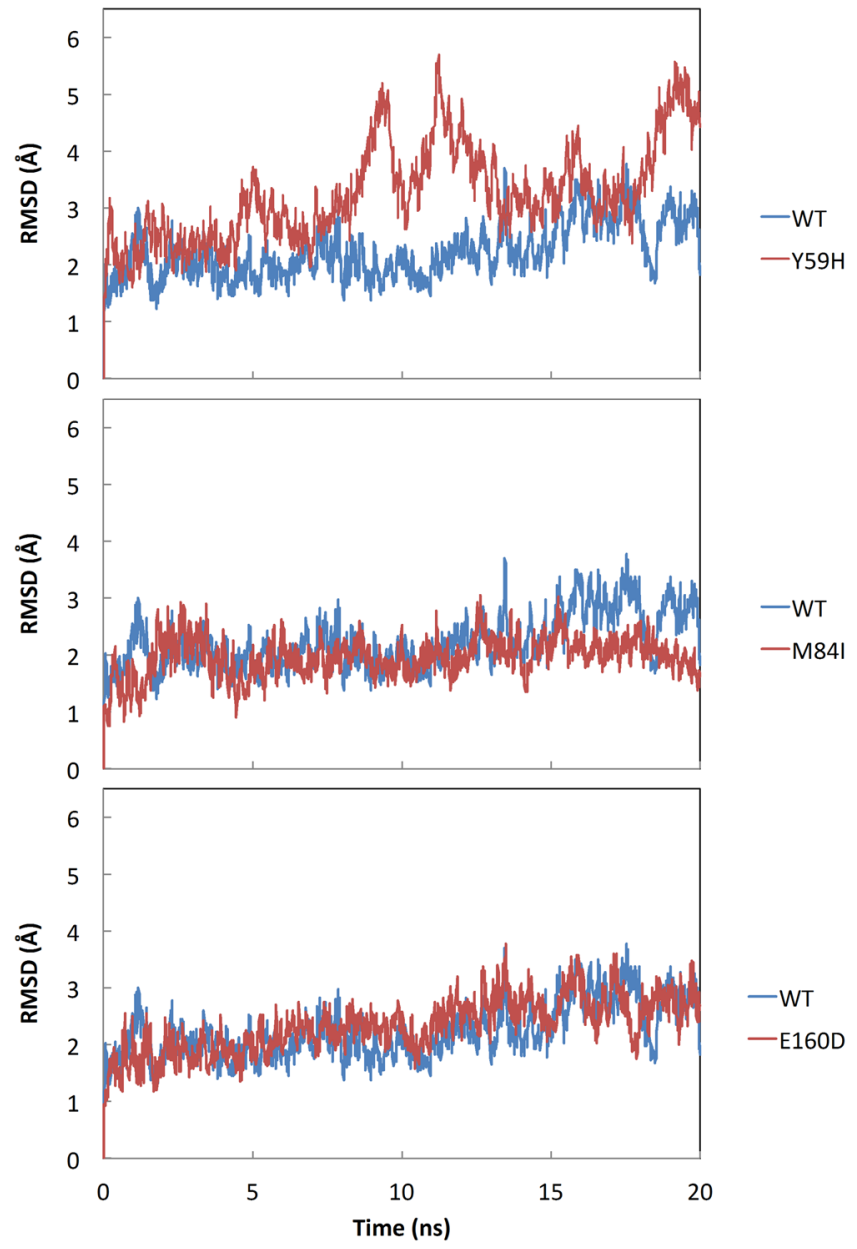

Figure 3. RMSD plot of the DBD. Comparison of the RMSD plots of the DBDs of WT and MT (A) Y59H, (B) M84I, and (C) E160D RamR structures with respect to the initial conformation during the course of the simulation. WT and MT plots are presented in blue and red, respectively.

WT-RamR structures for each C $\alpha$ atom (B-factor differences, df-B), followed by the mapping of the absolute values of df-B to the RamR structures (Fig. 4) for clear investigation of the fluctuation differences. The B-factor analysis demonstrated that the major fluctuation differences were at the $\mathrm{DBD}$, comprising most of the residues 
(A)

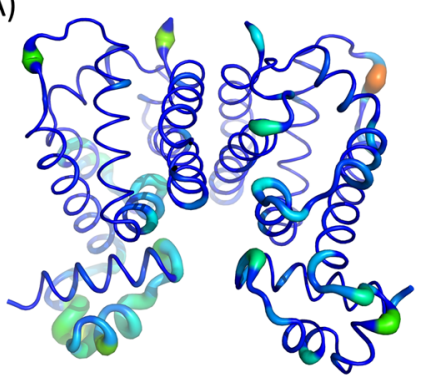

$\mathrm{Y} 59 \mathrm{H}$
(B)

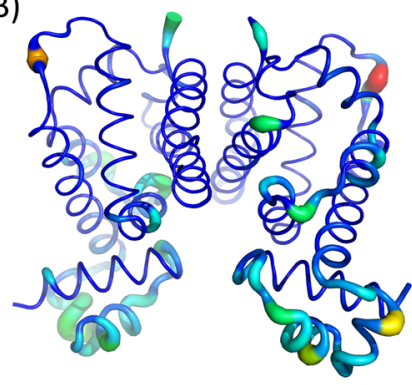

M84I
(C)

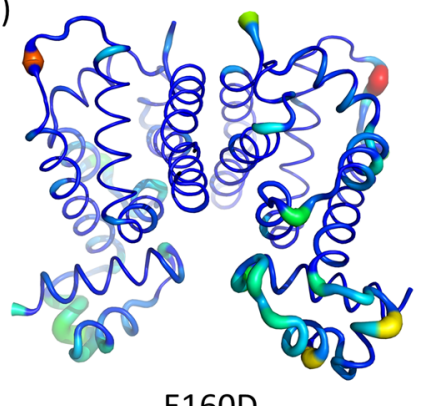

Figure 4. Analysis of the atomic fluctuations. The structures of (A) Y59H, (B) M84I, and (C) E160D RamR proteins are created as cartoon putty representations; blue and red represent the lowest and highest differences, respectively, of B-factor values compared with WT-RamR. In addition, the size of the tube reflects the differences in B-factor values; the larger the df-B values, the thicker the tube.

relevant to the DNA-binding functionality predicted in our RamR-DNA complex model, such as V32, R35, T43, and R46, for all three MT-RamR structures (Fig. 4).

RamR-DNA binding free energy. The RamR-DNA binding free energy differences between WT and MT structures were calculated in a series of alchemical free energy simulations and using the CGI protocol ${ }^{40}$. The DNA-binding affinity differences $\left(\Delta \Delta G_{\text {bind }}\right)$ were calculated according to $\Delta G_{1}$ (DNA-bound) $-\Delta G_{2}$ (DNA-free), as shown in the thermodynamic cycle (Fig. 5A). Notably, stabilizing mutations have negative $\Delta \Delta G$ values. We adopted the CGI protocol to calculate the binding affinity differences between DNA and RamR and the single point mutations. For DNA-free and DNA-bound structures, the free energy differences for $\mathrm{Y} 59 \mathrm{H}$ were $20.19 \pm 2.04$ and $21.41 \pm 1.59 \mathrm{~kJ} / \mathrm{mol}$ (Fig. 5B), for M84I were $25.03 \pm 0.92$ and $33.58 \pm 1.29 \mathrm{~kJ} / \mathrm{mol}$ (Fig. 5C), and for E160D were $-55.93 \pm 3.10$ and $-32.11 \pm 1.91 \mathrm{~kJ} / \mathrm{mol}$ (Fig. 5D), respectively. The resulting binding free energy differences $\left(\Delta \Delta G_{\text {bind }}\right)$ between WT- and Y59H-, M84I-, and E160D-RamR were 1.22, 8.55, and $23.82 \mathrm{~kJ} / \mathrm{mol}$, respectively (Fig. 5E). The DNA-binding affinity was disrupted in all mutants $\left(\Delta \Delta G_{\text {bind }}>0\right)$.

Normal mode analysis. Because normal mode analysis (NMA) is very time-consuming, we used the coarse-grained ENM ${ }^{46-48}$. The normal modes of WT-RamR and WT-RamR-DNA structures were calculated. For investigating the motional correlations between residues, we combined the 10 slowest modes to calculate the correlation heat maps for WT-RamR (Fig. 6A) and WT-RamR-DNA (Fig. 6B). The covariance matrix between residues represented by their $\mathrm{C} \alpha$ atoms was obtained from the ENM. The two covariance matrices indicated that the $\mathrm{N}$-terminal fragment $(\alpha 1-\alpha 7)$ differed markedly from the C-terminal fragment $(\alpha 8, \alpha 9)$ and showed increased levels of motional correlation (red, self-chain) and anticorrelation (blue, opposite chain) in the DNA-free RamR protein. This finding is consistent with the fluctuations calculated for the DNA-free RamR protein (Figure S1), which demonstrated that the amplitude of the fluctuations is larger than that in the RamR-DNA complex (Figure S2), particularly in the DNA-binding fragment. Compared with the DNA-free RamR protein, the RamRDNA complex exhibited increased levels of motional correlation either in the self-chains or opposite chains of the dimerization fragment $(\alpha 8, \alpha 9)$.

\section{Discussion}

The present study reveals that Y59H, M84I, and E160D mutations in the dimerization domain of RamR, which have been reported as MDR-relevant substitutions ${ }^{22,25,26}$, might affect RamR's binding affinity to the ramA promoter by altering the dynamics properties of the DBD. Previous study have suggested that amino acid residues Y59, M84, and E160 are considered to be involved in RamR substrate recognition and amino acid substitutions at these positions might reduce the DNA-binding affinity according to expand the distance between the N-terminal helix-turn-helix motifs in the $\mathrm{DBD}^{27}$. This inference could be also observed in our dynamics and binding free energy analysis, our studies demonstrate that these mutations could disrupt the RamR-DNA binding affinity through changing the dynamics networks of the RamR structure (Fig. 5).

The stability analysis and RMSD profiles reveal that the simulated Y59H-RamR structure exhibits obvious instability compared with the WT-RamR structure (Fig. 3). The Y59 residue is located at the junction of the $\mathrm{DBD}$ and the dimerization domain; therefore, a mutation at this position might affect the relevant collective motions relevant to DNA-binding functionality. The highly correlated motions of residue 59 and the DBD region (residues 2-42) can be observed in the correlation heat map (Fig. 6) that clearly demonstrate the influence of residue 59 mutated. The RMSF profile of $\mathrm{Y} 59 \mathrm{H}-\mathrm{RamR}$ shows that the fluctuations of the DBD region have obvious changes compared with WT-RamR (Figure S1). We mapped the fluctuation differences on the RamR structure and observed that the DBD region has obvious dynamics changes after the substitution of histidine for tyrosine at residue 59 (Fig. 4). The binding free energy calculations reveal that the $\mathrm{Y} 59 \mathrm{H}$ mutation may slightly disrupt the binding affinity of RamR to DNA (Fig. 5).

The RMSD profile of M84I-RamR revealed no influence on the structural stability compared with WT-RamR (Fig. 3). The RMSF profile of M84I-RamR show clear fluctuation changes in the DBD compared with that of WT-RamR (Figure S1). To visualize more clearly, the RMSF differences between WT- and M84I-RamR were 
(A)

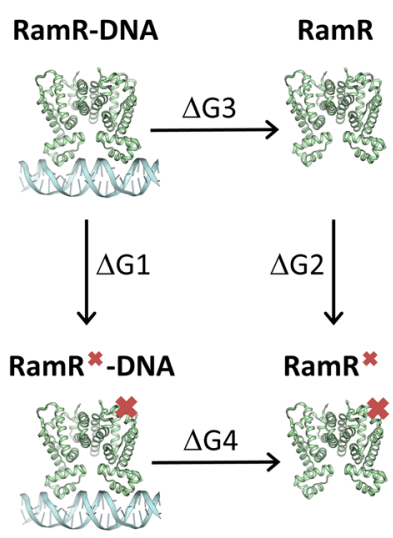

$(\mathrm{E})$

$$
\begin{aligned}
& \Delta \Delta G_{\text {bind }}=\Delta G 1-\Delta G 2 \\
& \Delta \Delta G^{{ }^{Y} 59 \mathrm{H}}=1.22 \mathrm{~kJ} / \mathrm{mol} \\
& \Delta \Delta G^{{ }^{M 84 I}}=8.55 \mathrm{~kJ} / \mathrm{mol} \\
& \Delta \Delta G^{\mathrm{E} 160 \mathrm{D}}=23.82 \mathrm{~kJ} / \mathrm{mol}
\end{aligned}
$$

(B)

B)

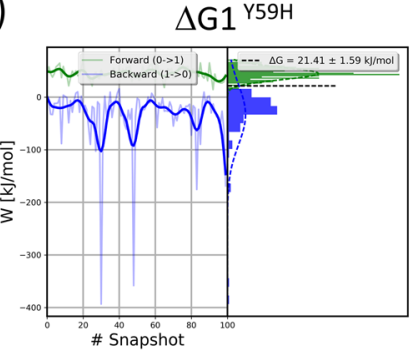

(C)

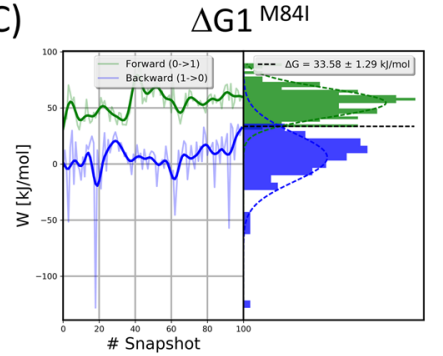

(D)

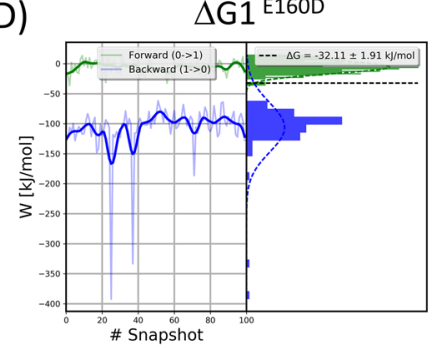

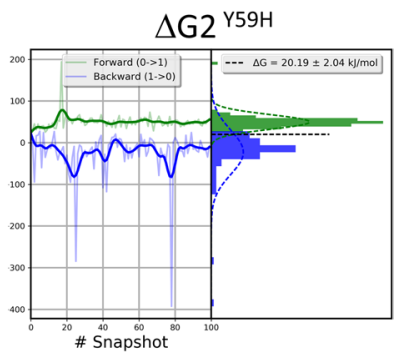
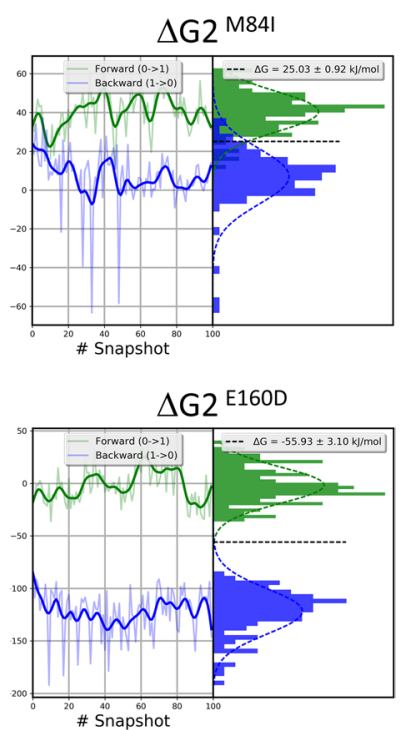

Figure 5. Analysis of the RamR-DNA binding free energy. (A) Thermodynamic cycle for binding affinity calculations. Distribution of the work values over time for an alchemical free energy simulation of (B) Y59H, (C) M84I, and (D) E160D mutations for the DNA-free (left panel) and DNA-bound (right panel) RamR proteins. The left plot in each result shows the work values obtained from the integration of $\delta H_{\lambda} / \delta \lambda$ as a function of the sampling time of the equilibrium states. The right plot in each result shows the histograms of work values from which the free energy was calculated. (E) In addition, the binding free energies caused by mutations $\left(\Delta \Delta G_{b i n d}\right)$ were calculated.

(A)

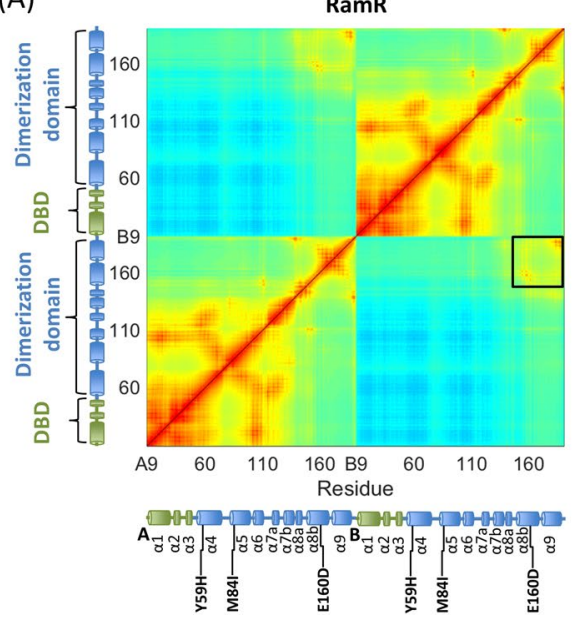

(B)
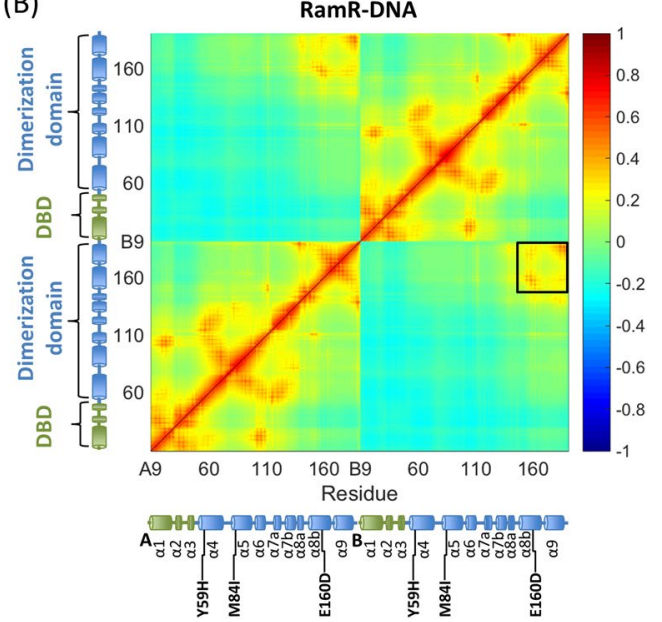

Figure 6. Cross-correlation map obtained from the ENM for the (A) DNA-free and (B) DNA-bound RamR proteins. A negative value refers to an anticorrelation between residue fluctuations (blue), whereas a positive value indicates concerted motion in the same direction (red). 
mapped to the M84I RamR structure to observe the more fluctuation changes occurring at the DBD region (Fig. 4). NMA reveal that because of the shear motion of the two domains, residue M84 forms a hinge (Fig. 6). The binding free energy calculations demonstrate that the M84I mutation may destabilize the RamR structure then disrupt the binding affinity of RamR to DNA (Fig. 5).

Residue 160 is located at the interface between the two monomers (Fig. 1). The cross-correlation maps (Fig. 6) indicate that the positive correlated motions are found in these regions (black squares) either in DNA-free or DNA-bound states of RamR. Therefore, mutation occurring at this position might strongly influence the dynamic networks of the RamR structure. However, meaningful differences were not observed between the RMSD, and RMSF profiles, possibly because the side chains are similar between the WT and the mutant (from glutamic acid to aspartic acid). The mutation free energy calculations also reveal that the E160D mutation may stabilize the RamR protein both in DNA-free and DNA-bound states $(\Delta G<0)$ (Fig. 5D) but this mutation does not contribute to increase the binding affinity of RamR to DNA $\left(\Delta \Delta G_{\text {bind }}>0\right)$ (Fig. 5E). The E160D mutated RamR has a tendency to the DNA-free form.

In addition, we also performed a simulation to analyze the RamR-RamR duplex dimerization free-energy changes upon these three mutations (Figure S3). For RamR monomer and dimer structures, the free energy differences for $\mathrm{Y} 59 \mathrm{H}$ were $19.49 \pm 1.15$ and $16.46 \pm 0.81 \mathrm{~kJ} / \mathrm{mol}$, for M84I were $14.29 \pm 0.86$ and $11.96 \pm 0.91 \mathrm{~kJ} / \mathrm{mol}$, and for E160D were $-36.78 \pm 0.92$ and $-14.24 \pm 1.05 \mathrm{~kJ} / \mathrm{mol}$, respectively (Figure S3). The resulting dimerization free-energy differences $\left(\Delta \Delta G_{\text {dimer }}\right)$ between WT- and Y59H-, M84I-, and E160D-RamR were -3.03, -2.33, and $22.54 \mathrm{~kJ} / \mathrm{mol}$, respectively (Figure S3). Compared with WT RamR, two mutants (Y59H and M84I) showed a slightly lower dimerization free energy for RamR dimer, suggesting that these mutations slightly increased the dimerization affinity of RmaR dimer (Figure S3), but these mutations do not contribute to increase the binding affinity of RamR to DNA $\left(\Delta \Delta G_{\text {bind }}>0\right)$ (Fig. 5). By contrast, mutants E160D showed a higher dimerization free energy, suggesting the decreased dimerization affinity of RmaR dimer. Interestingly, the position of E160D is just located at the binding interface of the RamR dimer. It is reasonable to assume that the reduction of the RamR-DNA binding affinity on E160D mutant is mainly caused by its instability in RamR duplex form.

\section{Conclusion}

In this study, we have applied computational methods to understand the structural implications of Y59H, M84I, and E160D in RamR that are related to induce bacterial drug resistance. Our findings underscore that the Y59H, M84I, and E160D mutations may exhibit different behavior as compared to RamR WT, especially in DNA binding domain. In summary, our simulated results can properly in accordance with current study results, which demonstrated that amino acid substitutions of Y59H, M84I, and E160D in RamR might reduce its DNA-binding affinity.

\section{Data Availability}

All data generated or analysed during this study are included in this published article (and its Supplementary Information files).

\section{References}

1. Nordmann, P., Cuzon, G. \& Naas, T. The real threat of Klebsiella pneumoniae carbapenemase-producing bacteria. Lancet Infect Dis 9, 228-236, https://doi.org/10.1016/S1473-3099(09)70054-4 (2009).

2. Gupta, N., Limbago, B. M., Patel, J. B. \& Kallen, A. J. Carbapenem-resistant Enterobacteriaceae: epidemiology and prevention. Clin Infect Dis 53, 60-67, https://doi.org/10.1093/cid/cir202 (2011).

3. Tenover, F. C. Mechanisms of antimicrobial resistance in bacteria. Am J Med 119, S3-10; discussion S62-70, https://doi. org/10.1016/j.amjmed.2006.03.011 (2006)

4. Sun, J., Deng, Z. \& Yan, A. Bacterial multidrug efflux pumps: mechanisms, physiology and pharmacological exploitations. Biochem Biophys Res Commun 453, 254-267, https://doi.org/10.1016/j.bbrc.2014.05.090 (2014).

5. Nikaido, E., Yamaguchi, A. \& Nishino, K. AcrAB multidrug efflux pump regulation in Salmonella enterica serovar Typhimurium by RamA in response to environmental signals. J Biol Chem 283, 24245-24253, https://doi.org/10.1074/jbc.M804544200 (2008).

6. Yu, E. W., Aires, J. R. \& Nikaido, H. AcrB multidrug efflux pump of Escherichia coli: composite substrate-binding cavity of exceptional flexibility generates its extremely wide substrate specificity. J Bacteriol 185, 5657-5664 (2003).

7. Costa, S. S., Viveiros, M., Amaral, L. \& Couto, I. Multidrug Efflux Pumps in Staphylococcus aureus: anUpdate. Open Microbiol J. 7, 59-71, https://doi.org/10.2174/1874285801307010059 (2013).

8. Padilla, E. et al. Klebsiella pneumoniae AcrAB efflux pump contributes to antimicrobial resistance and virulence. Antimicrobial agents and chemotherapy 54, 177-183, https://doi.org/10.1128/AAC.00715-09 (2010).

9. Webber, M. A. \& Piddock, L. J. The importance of efflux pumps in bacterial antibiotic resistance. The Journal of antimicrobial chemotherapy 51, 9-11 (2003).

10. Nikaido, H. \& Takatsuka, Y. Mechanisms of RND multidrug efflux pumps. Biochim Biophys Acta 1794, 769-781, https://doi. org/10.1016/j.bbapap.2008.10.004 (2009).

11. Pao, S. S., Paulsen, I. T. \& Saier, M. H. Jr. Major facilitator superfamily. Microbiol Mol Biol Rev 62, 1-34 (1998).

12. Dean, M., Rzhetsky, A. \& Allikmets, R. The human ATP-binding cassette (ABC) transporter superfamily. Genome Res $\mathbf{1 1}$, 1156-1166, https://doi.org/10.1101/gr.184901 (2001).

13. Bay, D. C., Rommens, K. L. \& Turner, R. J. Small multidrug resistance proteins: a multidrug transporter family that continues to grow. Biochim Biophys Acta 1778, 1814-1838, https://doi.org/10.1016/j.bbamem.2007.08.015 (2008).

14. Magalhaes, J. V. et al. A gene in the multidrug and toxic compound extrusion (MATE) family confers aluminum tolerance in sorghum. Nat Genet 39, 1156-1161, https://doi.org/10.1038/ng2074 (2007)

15. Fernando, D. M. \& Kumar, A. Resistance-Nodulation-Division Multidrug Efflux Pumps in Gram-Negative Bacteria: Role in Virulence. Antibiotics (Basel) 2, 163-181, https://doi.org/10.3390/antibiotics2010163 (2013).

16. Du, D. et al. Structure of the AcrAB-TolC multidrug efflux pump. Nature 509, 512-515, https://doi.org/10.1038/nature13205 (2014).

17. George, A. M., Hall, R. M. \& Stokes, H. W. Multidrug resistance in Klebsiella pneumoniae: a novel gene, ramA, confers a multidrug resistance phenotype in Escherichia coli. Microbiology 141(Pt 8), 1909-1920, https://doi.org/10.1099/13500872-141-8-1909 (1995).

18. Chollet, R., Chevalier, J., Bollet, C., Pages, J. M. \& Davin-Regli, A. RamA is an alternate activator of the multidrug resistance cascade in Enterobacter aerogenes. Antimicrobial agents and chemotherapy 48, 2518-2523, https://doi.org/10.1128/AAC.48.7.2518-2523.2004 (2004). 
19. van der Straaten, T., Janssen, R., Mevius, D. J. \& van Dissel, J. T. Salmonella gene rma (ramA) and multiple-drug-resistant Salmonella enterica serovar typhimurium. Antimicrobial agents and chemotherapy 48, 2292-2294, https://doi.org/10.1128/AAC.48.6.22922294.2004 (2004).

20. Rosenblum, R., Khan, E., Gonzalez, G., Hasan, R. \& Schneiders, T. Genetic regulation of the ramA locus and its expression in clinical isolates of Klebsiella pneumoniae. Int J Antimicrob Agents 38, 39-45, https://doi.org/10.1016/j.ijantimicag.2011.02.012 (2011).

21. Veleba, M. \& Schneiders, T. Tigecycline resistance can occur independently of the ramA gene in Klebsiella pneumoniae. Antimicrobial agents and chemotherapy 56, 4466-4467, https://doi.org/10.1128/AAC.06224-11 (2012).

22. Abouzeed, Y. M., Baucheron, S. \& Cloeckaert, A. ramR mutations involved in efflux-mediated multidrug resistance in Salmonella enterica serovar Typhimurium. Antimicrobial agents and chemotherapy 52, 2428-2434, https://doi.org/10.1128/AAC.00084-08 (2008).

23. Ricci, V. \& Busby, S. J. \& Piddock, L. J. Regulation of RamA by RamR in Salmonella enterica serovar Typhimurium: isolation of a RamR superrepressor. Antimicrobial agents and chemotherapy 56, 6037-6040, https://doi.org/10.1128/AAC.01320-12 (2012).

24. Hentschke, M., Wolters, M., Sobottka, I., Rohde, H. \& Aepfelbacher, M. ramR mutations in clinical isolates of Klebsiella pneumoniae with reduced susceptibility to tigecycline. Antimicrobial agents and chemotherapy 54, 2720-2723, https://doi.org/10.1128/ AAC.00085-10 (2010).

25. Kehrenberg, C., Cloeckaert, A., Klein, G. \& Schwarz, S. Decreased fluoroquinolone susceptibility in mutants of Salmonella serovars other than Typhimurium: detection of novel mutations involved in modulated expression of ramA and soxS. J Antimicrob Chemoth 64, 1175-1180, https://doi.org/10.1093/jac/dkp347 (2009).

26. Akiyama, T. \& Khan, A. A. Molecular characterization of strains of fluoroquinolone-resistant Salmonella enterica serovar Schwarzengrund carrying multidrug resistance isolated from imported foods. The Journal of antimicrobial chemotherapy 67, 101-110, https://doi.org/10.1093/jac/dkr414 (2012).

27. Yamasaki, S. et al. The crystal structure of multidrug-resistance regulator RamR with multiple drugs. Nature communications 4, 2078, https://doi.org/10.1038/ncomms3078 (2013).

28. Eswar, N. et al. Comparative protein structure modeling using Modeller. Current protocols in bioinformatics Chapter 5, Unit5 6, https://doi.org/10.1002/0471250953.bi0506s15 (2006).

29. Laskowski, R. A., Macarthur, M. W., Moss, D. S. \& Thornton, J. M. PROCHECK - A program to check the stereochemical quality of protein structures. Journal of Applied Crystallography 26, 283-291 (1993).

30. Arfken, G. The Method of Steepest Descents. \$7.4 in Mathematical Methods for Physicists, 3rd ed Orlando: Academic Press, $428-436$ (1985).

31. PyMOL. Available: http://www.pymol.org. Accessed (2017).

32. Baucheron, S. et al. Binding of the RamR repressor to wild-type and mutated promoters of the ramA gene involved in effluxmediated multidrug resistance in Salmonella enterica serovar Typhimurium. Antimicrobial agents and chemotherapy 56, 942-948, https://doi.org/10.1128/AAC.05444-11 (2012)

33. van Dijk, M. \& Bonvin, A. M. 3D-DART: a DNA structure modelling server. Nucleic acids research 37, W235-239, https://doi. org/10.1093/nar/gkp287 (2009).

34. Dominguez, C., Boelens, R. \& Bonvin, A. M. J. J. HADDOCK: A protein-protein docking approach based on biochemical or biophysical information. J Am Chem Soc 125, 1731-1737, https://doi.org/10.1021/ja026939x (2003).

35. van Zundert, G. C. P. et al. The HADDOCK2.2 Web Server: User-Friendly Integrative Modeling of Biomolecular Complexes. Journal of molecular biology 428, 720-725, https://doi.org/10.1016/j.jmb.2015.09.014 (2016).

36. Van Der Spoel, D. et al. GROMACS: fast, flexible, and free. Journal of computational chemistry 26, 1701-1718, https://doi. org/10.1002/jcc.20291 (2005).

37. Hess, B., Bekker, H., Berendsen, H. J. C. \& Fraaije, J. LINCS: A linear constraint solver for molecular simulations. Journal of computational chemistry 18, 1463-1472 (1997).

38. Gapsys, V., Michielssens, S., Seeliger, D. \& de Groot, B. L. pmx: Automated protein structure and topology generation for alchemical perturbations. Journal of computational chemistry 36, 348-354, https://doi.org/10.1002/jcc.23804 (2015).

39. Gapsys, V. \& de Groot, B. L. pmx Webserver: A User Friendly Interface for Alchemistry. Journal of chemical information and modeling 57, 109-114, https://doi.org/10.1021/acs.jcim.6b00498 (2017).

40. Goette, M. \& Grubmuller, H. Accuracy and convergence of free energy differences calculated from nonequilibrium switching processes. Journal of computational chemistry 30, 447-456, https://doi.org/10.1002/jcc.21073 (2009).

41. Brooks, B. \& Karplus, M. Harmonic dynamics of proteins: normal modes and fluctuations in bovine pancreatic trypsin inhibitor. Proceedings of the National Academy of Sciences of the United States of America 80, 6571-6575 (1983).

42. Levitt, M., Sander, C. \& Stern, P. S. Protein normal-mode dynamics: trypsin inhibitor, crambin, ribonuclease and lysozyme. Journal of molecular biology 181, 423-447 (1985).

43. Kidera, A. \& Go, N. Normal mode refinement: crystallographic refinement of protein dynamic structure. I. Theory and test by simulated diffraction data. Journal of molecular biology 225, 457-475 (1992).

44. Tama, F. \& Brooks, C. L. Symmetry, form, and shape: guiding principles for robustness in macromolecular machines. Annual review of biophysics and biomolecular structure 35, 115-133, https://doi.org/10.1146/annurev.biophys.35.040405.102010 (2006).

45. Thomas, A., Hinsen, K., Field, M. J. \& Perahia, D. Tertiary and quaternary conformational changes in aspartate transcarbamylase: a normal mode study. Proteins 34, 96-112 (1999).

46. Tirion, M. M. Large Amplitude Elastic Motions in Proteins from a Single-Parameter, Atomic Analysis. Physical review letters 77, 1905-1908, https://doi.org/10.1103/PhysRevLett.77.1905 (1996).

47. Bahar, I., Atilgan, A. R. \& Erman, B. Direct evaluation of thermal fluctuations in proteins using a single-parameter harmonic potential. Folding \& design 2, 173-181, https://doi.org/10.1016/S1359-0278(97)00024-2 (1997).

48. Ming, D., Kong, Y., Lambert, M. A., Huang, Z. \& Ma, J. How to describe protein motion without amino acid sequence and atomic coordinates. Proceedings of the National Academy of Sciences of the United States of America 99, 8620-8625, https://doi.org/10.1073/ pnas.082148899 (2002).

49. Straube, J., Huang, B. E. \& Cao, K. L. DynOmics to identify delays and co-expression patterns across time course experiments. Scientific reports 7, 40131, https://doi.org/10.1038/srep40131 (2017).

\section{Acknowledgements}

We are grateful to Institute of Medical Science and Technology, National Sun Yat-sen University for providing the hardware and software resources. This study was mainly supported by the grant from 'Academic Summit Program' of Ministry of Science and Technology (MOST-105-2221-E-110-079), NSYSU-KMU joint research project, (\#NSYSUKMU 106-I006), and 'Medical Science and Technology Center of Aiming for the Top University Program' of National Sun Yat-sen University and Ministry of Education, Taiwan.

\section{Author Contributions}

Conceived and designed the experiments: Y.Y.L. and C.C.C. Performed the experiments and analyzed the data: Y.Y.L. and C.C.C. Contributed materials/analysis tools and wrote the paper: Y.Y.L. and C.C.C. 


\section{Additional Information}

Supplementary information accompanies this paper at https://doi.org/10.1038/s41598-017-14008-5.

Competing Interests: The authors declare that they have no competing interests.

Publisher's note: Springer Nature remains neutral with regard to jurisdictional claims in published maps and institutional affiliations.

(c) (1) Open Access This article is licensed under a Creative Commons Attribution 4.0 International License, which permits use, sharing, adaptation, distribution and reproduction in any medium or format, as long as you give appropriate credit to the original author(s) and the source, provide a link to the Creative Commons license, and indicate if changes were made. The images or other third party material in this article are included in the article's Creative Commons license, unless indicated otherwise in a credit line to the material. If material is not included in the article's Creative Commons license and your intended use is not permitted by statutory regulation or exceeds the permitted use, you will need to obtain permission directly from the copyright holder. To view a copy of this license, visit http://creativecommons.org/licenses/by/4.0/.

(C) The Author(s) 2017 